\title{
Evaluación de la calidad en la educación básica y media en Colombia
}

\section{Assessment of quality in basic and medium education in Colombia}

DOI: http://dx.doi.org/10.17981/cultedusoc.11.2.2020.08

Recibido: 24 de diciembre de 2019 Aceptado: 25 de marzo de 2020 Publicado: 07 de julio de 2020

Jhan Carlos Herrera Pérez

IED Reuven Feuerstein. Barranquilla (Colombia)

stickmaster2005@gmail.com

Para citar este artículo:

Herrera J. (2020). Evaluación de la calidad en la educación básica y media en Colombia. Cultura, Educación y Sociedad, 11(2). 125-144. DOI: http://dx.doi.org/10.17981/cultedusoc.11.2.2020.08

\section{Resumen}

El presente artículo tiene como objetivo analizar algunas acciones emprendidas por el MEN (Ministerio de Educación Nacional) para alcanzar la calidad educativa en Colombia. Se utiliza una metodología de tipo cualitativo, la técnica de análisis documental y como instrumento una ficha de registro de datos; se consultaron fuentes como artículos y tesis actualizadas sobre la temática, destacando las categorías de análisis: concepciones y generalidades de la educación en Colombia, el impacto de la calidad educativa en una nación, la evaluación de los aprendizajes en los estudiantes, el problema de las desigualdades entre escuelas. Los resultados muestran que el MEN, ha implementado diferentes pruebas en el ámbito educativo del estudiante (SABER $3^{\circ}, 5^{\circ}, 9^{\circ}, 11^{\circ}$ ), programas para el fortalecimiento de la formación docente como PTA (Todos a Aprender) y Modelo Singapur, así como fomentar la lectura y escritura a nivel nacional a través del programa "Leer es mi cuento". Las conclusiones develan que pese a los esfuerzos realizados por el gobierno nacional, los resultados obtenidos en las pruebas nacionales e internacionales PISA no se reflejan grandes avances, por lo que indudablemente se requiere mejorar la selección docente, su remuneración y atraer a los mejores a las facultades de educación, se tienen que adaptar las estrategias pedagógicas en educación, asimismo, implementar medidas como la jornada única en todas las escuelas de la nación, ofreciendo todas las garantías a nivel de alimentación, infraestructura escolar, entre otros que facilitan la labor docente en el proceso de enseñanza-aprendizaje de los estudiantes.

Palabras clave: Calidad de la educación; evaluación de la educación; formación de docentes; pruebas estandarizadas

\begin{abstract}
This article aims to analyze some actions taken by the MEN (Ministry of National Education) to achieve educational quality in Colombia. A qualitative methodology is used, the documentary analysis technique and as an instrument a data registration form; sources such as updated articles and theses on the subject were consulted, highlighting the categories of analysis: conceptions and generalities of education in Colombia, the impact of educational quality in a nation, the evaluation of student learning, the problem of inequalities between schools. The results show that the MEN has implemented different tests in the student's educational environment (KNOW 3rd, 5th, 9th, 11th), programs to strengthen teacher training such as PTA (All to Learn) and the Singapore Model , as well as promoting reading and writing nationwide through the program "Reading is my story." The conclusions reveal that despite the efforts made by the national government, the results obtained in the national and international PISA tests do not reflect great progress, so there is undoubtedly a need to improve teacher selection, remuneration and attract the best to the faculties of education, they must adapt the pedagogical strategies in education, also, implement measures such as the single day in all the schools of the nation, offering all the guarantees at the level of food, school infrastructure, among others that facilitate the teaching work in the teaching-learning process of the students.
\end{abstract}

Keywords: Quality of education; education evaluation; teacher training; standardized tests 


\section{INTRODUCCIÓN}

El objetivo del artículo es realizar un análisis sobre algunas acciones emprendidas por el MEN (Ministerio de Educación Nacional) para alcanzar la calidad educativa en Colombia. Así pues, es importante evaluar algunas iniciativas, los programas que se están llevando a cabo por este ministerio, cuales están siendo eficaces, las problemáticas que surgen del contexto de los estudiantes para alcanzar los objetivos en su proceso educativo y mostrar la importancia de realizar grandes esfuerzos en términos económicos a este sector por el impacto positivo que tiene en la nación en el mediano y largo plazo.

Hablar de evaluación es involucrar un sinnúmero de actores que hacen parte del proceso educativo, entre los cuales están los estudiantes, docentes, padres de familia, directivos docentes, secretarios de educación, ministros de educación, que sin lugar a duda colocan su mayor esfuerzo, aportes, que conlleven a la obtención de mejores resultados en las pruebas nacionales e internacionales, además de la formación integral del estudiante.

Si bien es cierto son significativos los esfuerzos que el conjunto de los países de la región incluyendo Colombia, han venido desarrollando en relación a instalar sistemas nacionales y subnacionales de evaluación educativa, aún resultan insuficientes en términos de proporcionar insumos efectivos, que orienten el desarrollo de políticas educativas para el mejoramiento de la calidad de la educación (Organización de las Naciones Unidas para la Educación, la Ciencia y la Cultura, UNESCO, 2008, p. 6)

Las pruebas nacionales e internacionales estandarizadas, si bien, son un referente para determinar las problemáticas de determinados sectores de la sociedad a nivel local o global, no permiten tener una visión general de la educación que reciben nuestros niños, ya que no los evalúan en todas sus dimensiones y no se tienen en cuenta aspectos característicos de una población determinada, lo cual los hace únicos y los distingue de todos los demás. Sin embargo, miden ciertos criterios que son relevantes para implementar las reformas educativas en los países y que permitan obtener mejores resultados, mejorando el proceso educativo y que este logre apalancar la dinámica con que se están moviendo todos los sectores de la sociedad y que conlleven al progreso social, económico, etc.

Es importante que el gobierno colombiano, genere políticas de Estado, que sean perdurables en el tiempo, ya que los resultados en educación se observan a mediano y largo plazo, las pruebas estandarizadas como PISA, deben servir para tomar decisiones sobre las políticas educativas y las reformas pertinentes que deberán implementarse para ser más competentes en este mundo globalizado, pero sin dejar de lado el aspecto formativo, además de reflexionar sobre la forma en cómo se está evaluando internamente a través de su sistema de evaluación nacional, hacer los ajustes necesarios y encaminarse en la dinámica que el mundo le está exigiendo en la actualidad.

Es así como esta revisión sistemática, permite reconocer algunas iniciativas del gobierno nacional para mejorar la calidad educativa, contrastar algunas ventajas y desventajas en las diferentes instituciones educativas y el rezago de estas en el área rural, analizar la incidencia de la formación docente en los resultados de los estudiantes en las pruebas nacionales e internacionales, además de señalar la importancia de trabajar en conjunto todos los actores que intervienen en el proceso educativo en la búsqueda de mejores resultados en el mediano y largo plazo. 
Con base en estas consideraciones, a lo largo de este artículo, se abordará uno de los temas más notables en el sector de la educación, como lo es la evaluación de la calidad educativa en Colombia, una mirada acerca de las evaluaciones nacionales e internacionales para medir las competencias de los estudiantes, presentar y analizar algunas problemáticas que enfrenta la sociedad y su repercusión por los resultados obtenidos, la importancia del docente y algunos de los retos para mejorar la calidad educativa en el país.

\section{Metodología}

Se utilizó una metodología de tipo cualitativo, la técnica de análisis documental y como instrumento de recolección de datos se diseñó una ficha de registro de datos, se realizó una revisión sistemática de artículos y tesis actualizadas, el análisis considera las siguientes categorías: concepciones y generalidades de la educación en Colombia, el impacto de la calidad educativa en una nación, la evaluación de los aprendizajes en los estudiantes, el problema de las desigualdades entre escuelas. Se realizó una búsqueda bibliográfica con esos descriptores en Google Académico, Redalyc, Dialnet y Scielo, los registros oscilaron entre 90 y 110 registros, se seleccionaron los de mayor relevancia de acuerdo a una lectura crítica de los documentos y teniendo en cuenta los más actualizados al tema en cuestión.

Luego se realizó una tabla en el programa Microsoft Office Word en donde se organizó la información de cada uno de los documentos seleccionados, la cual permitió comparar los resultados de cada estudio, sus similitudes y diferencias, hallazgos y demás información importante de los mismos. Esta rejilla tiene aspectos como título, autor, año de publicación, revista, objetivos, tipo de estudio, muestra, resultados y conclusiones. Se determinó después de un análisis que la muestra está compuesta por 44 documentos que en su gran mayoría son artículos. Se descartaron aquellos que no están directamente relacionados con las categorías de análisis e igualmente los que proporcionaban información redundante.

En el criterio de búsqueda se tuvo en consideración documentos del año 2000 hasta el 2020 y en menor medida algunos anteriores de este rango fecha por su importancia para la investigación. Este proceso de búsqueda se ha llevado a cabo durante el periodo comprendido entre junio de 2019 y junio de 2020, se hizo tanto en español como en inglés. Se privilegiaron aquellos textos que provenían de entidades como la OCDE (Organización para la Cooperación y el Desarrollo Económicos), MEN (Ministerio de Educación Nacional), Sindicato de maestros (FECODE), artículos de docentes y rectores del país.

\section{Resultados y Discusión}

\section{Concepciones y generalidades de la educación en Colombia}

En este apartado se hará un acercamiento sobre cómo está organizada la educación en Colombia, que tipos de evaluación se practican a nivel nacional e internacional, las entidades que aplican dichas pruebas y que instrumentos utilizan, así como las características de la profesión docente, su vinculación, desempeño, formación y el programa PTA para afianzar las practicas educativas en las instituciones de más bajo desempeño en las pruebas nacionales. 
La educación se puede concebir como un proceso en el que intervienen diversos actores, desde los que hacen parte de la familia, hasta los individuos que hacen parte de su contexto, es allí donde el individuo comienza a aprender en la socialización con el medio que lo rodea y de las experiencias, luego en la escuela se afianzan las relaciones con personas de su mismo rango de edades y además se incorporan una serie de conocimientos que se vinculan a los que ya posee en su estructura cognitiva. Es importante entonces, que todos los responsables de la educación estén asegurándose a través de un seguimiento, que los niños están aprendiendo de forma eficiente, en este sentido, Arancibia (1997) expresa que: "deben evitarse los aprendizajes de mala calidad y para ello hay que tener una serie de controles que permita corregir los defectos y que los jóvenes aprendan de acuerdo con sus necesidades y expectativas de la sociedad" (p. 4).

De acuerdo a lo anterior, es imprescindible que desde el gobierno nacional se generen políticas educativas que giren en torno a conseguir la calidad educativa en todo el territorio nacional, tanto en las instituciones de carácter público, como privadas, desde lo urbano hasta lo rural. Para ello, tiene que priorizar recursos para este sector y dejar de verlo como un gasto cuando en realidad es una inversión, luego de esto, generar herramientas, programas, designar responsables y hacer seguimiento, para que al final los estudiantes obtengan buenos resultados académicos y se formen como ciudadanos con valores.

Colombia es un estado unitario, social de derecho; con 32 departamentos descentralizados y un distrito capital que aloja la sede del gobierno nacional. Según el Departamento Administrativo Nacional de Estadística (DANE, 2018), Colombia alcanza los 48.258.494 de habitantes, siendo uno de los países latinoamericanos, junto a Brasil, y México con más habitantes. De esta población, la mayor parte se concentra en las zonas urbanas $77.1 \%$ y un $22,9 \%$ en zonas rurales. Con las diferentes reformas que ha tenido el sector educativo colombiano, se ha logrado avanzar significativamente en materia de cobertura. Al respecto la Organización para la Cooperación y Desarrollo Económico (OECD) sostiene:

Las tasas de matrícula han incrementado en la educación media e inicial, y Colombia también ha progresado en cuanto a la expansión del acceso a la educación y el cuidado de la primera infancia, así como también a la educación superior. Sin embargo, aún necesita incrementar la cobertura, mantener a los estudiantes en la escuela y suavizar las transiciones. Las brechas en materia de tasas de matriculación siguen existiendo entre las áreas urbanas y rurales y entre los estudiantes desfavorecidos y favorecidos, particularmente en la educación preescolar y la educación media (OECD, 2018, p. 8)

Con la aparición del coronavirus, no solo el gobierno colombiano sino mundial, tendrán que realizar estudios sobre el impacto de la pandemia en cuanto a la tasa de deserción escolar, en mayor medida en el sector rural donde no se cuentan con los recursos tecnológicos para seguir con las clases de forma virtual, al igual que muchos estudiantes del área urbana de estratos bajos en Colombia.

Ahora bien, el gobierno nacional ha avanzado significativamente en el tema cobertura educativa, aunque hay aspectos que mejorar como la relación número de estudiantesdocentes por curso, ya que esto permite conocer mejor a los estudiantes en cuanto a sus necesidades de aprendizajes, reconocer de manera más fácil sus destrezas, habilidades, competencias, que contribuyen de manera significativa al mejoramiento de la calidad educativa, al respecto, se encuentra un punto muy importante que es el de la evaluación, 
es así como (Black \& Wiliam, 1998; Córdoba, 2006; Díaz-Barriga y Hernández, 2002; Crooks, 1988) afirman que: existe un consenso generalizado en cuanto a que no es posible mejorar la calidad de la educación sin considerar la evaluación, que no debe confundirse con calificación, para ello deben tenerse en cuenta diversos aspectos del estudiante, es decir la evaluación debe ser integral y formativa.

Las organizaciones y los gobiernos realizan pruebas que permiten la medición del conocimiento, destrezas y habilidades de los estudiantes y de paso la calidad de la educación de un país, sobre dichas pruebas, Braun y Kanjee (2006) consideran que se emiten juicios que permiten hacer una evaluación y posterior valoración de los resultados. En nuestro país se realizan pruebas nacionales a los estudiantes a lo largo de su vida escolar.

En Colombia, a nivel nacional, la educación en general es sometida a pruebas y es el ICFES, según el Ministerio de Educación Nacional la entidad responsable de la evaluación de la educación colombiana y para ello realiza la valoración de la calidad de la educación básica -pruebas SABER, aplicadas periódicamente a estudiantes de tercero, quinto y noveno grados (Campos y Fajardo, 2015, p. 4)

Así mismo, "el Instituto Colombiano para la Evaluación de la Educación (ICFES) tiene a su cargo los exámenes de Estado de la educación media (SABER 11.), que desde 1980 es obligatorio" (ICFES, 2012, p. 7) y de la educación superior (SABER PRO). Por otro lado, también se realizan pruebas externas internacionales (MEN, 2009), con el fin de conocer el impacto de sus políticas en educación Básica y Media, Colombia participa en tres pruebas: PISA, SERCE y TERCE (Segundo y Tercer Estudio Comparativo Explicativo Regional), TIMSS (Estudio Internacional de Tendencias en Matemáticas y Ciencias) (Red Latinoamericana por la Educación, REDUCA, 2015).

De las evidencias anteriores, se puede decir que en el país se han implementado una serie de pruebas para medir cómo va la educación, además de hacer parte de organismos que apoyan la implementación de pruebas estandarizadas y de acuerdo a los resultados obtenidos tomar medidas para ajustar las políticas educativas. Sin embargo, después de muchos esfuerzos realizados, la OECD (2016) sostiene que "a pesar de transformar significativamente su sistema de educación durante las últimas dos décadas, Colombia enfrenta dos desafíos críticos: altos niveles de desigualdad desde los primeros años de educación y un bajo nivel de calidad en el sistema educativo" (p. 1).

Por otro lado, hay algunos autores que expresan lo contrario, como Ayala-García (2015), quien afirma que "los bajos resultados que Colombia ha obtenido en evaluaciones nacionales e internacionales permiten afirmar que los esfuerzos no han sido suficientes para alcanzar los estándares deseados" (p. 4).

Avanzar en aspectos como la universalización de la educación preescolar, las diferencias sustanciales en cuanto a cobertura y calidad educativa entre el sector rural y urbano, así como el fortalecimiento de la educación media, son unas de las grandes apuestas del gobierno nacional en los últimos años, partiendo desde el punto de vista que la educación es el más poderoso instrumento de igualdad social. En MEN (2016), se plantean algunas líneas estratégicas para la consecución de algunos de estos objetivos como la Jornada Única, Excelencia Docente, más acceso a la educación superior de calidad, entre otras. 
Alrededor de estas políticas se impulsan programas y estrategias que permitan alcanzar la calidad educativa, algunas de ellas son: el programa Todos a aprender, para mejorar los resultados en las áreas de matemáticas y lenguaje; el Día de la Excelencia Educativa, en el que la comunidad educativa de las diferentes instituciones del país reflexionan en torno a la importancia de la calidad de la educación; el Índice Sintético de Calidad Educativa, que plantea metas a cada establecimiento educativo para mejorar la educación; y la implementación del programa de alimentación escolar para mejorar la calidad educativa (Chacón, 2019, p. 40).

El MEN diseña una herramienta para el seguimiento del progreso de las instituciones educativas, denominado ISCE (Índice Sintético de Calidad Educativa), el cual permite identificar las fortalezas que se tienen y en qué áreas se debe mejorar. Existen algunos problemas con esta escala a través de la cual se mide la calidad educativa en Colombia tales como lo expresa Zambrano (2015):

I) Se muestra como el principal parámetro de evaluación, II) no ha sido claro con los grupos poblacionales hacía los cuáles va dirigido (se supone que va dirigido a las instituciones educativas, pero se le entregó a todo el país en un ejercicio publicitario que tal vez buscaba incrementar la popularidad del MEN), III) desconoce la diversidad y condiciones particulares de las instituciones educativas, IV) no integra diferentes concepciones de calidad, es más un indicador de productividad académica con la intención de reforzar lógicas fabriles al interior de la escuela, V) aparece como una medición de calidad unidireccional y arbitraria, VI) presenta problemas con la selección de los indicadores, VII) no registra las deficiencias y carencias de las instituciones como un insumo de entrada y VIII) no presenta acompañamiento de instituciones independientes del gobierno y acreditadas por instancias internacionales, lo cual deja pendiente el tema de la transparencia (p. 13).

Algunos de los problemas asociados a la calidad de la educación se pueden destacar el mejoramiento de los contenidos curriculares, material didáctico y formación, capacitación y actualización de docentes. En Camargo (2001), señala similares argumentos para lograr un verdadero proceso de enseñanza-aprendizaje y que "fueron expuestos en una reunión en el año 1982 por integrantes de la OEA-Organización de Estados Americanos-" (p. 4).

De lo anteriormente expuesto, se puede resaltar el papel que juega el docente en la consecución de una calidad educativa a niveles óptimos, es por ello que debe tenerse en cuenta quienes ingresan a esta profesión, la forma en que el gobierno a través del ministerio de educación capacita a los docentes para mejorar en sus prácticas educativas, entre otras. En este sentido Robalino (2005) afirma:

Desde esta perspectiva, supone abrirse al reconocimiento que hay un conjunto de factores que determinan el desempeño. Entre ellos: formación inicial, desarrollo profesional en servicio, condiciones de trabajo, salud, autoestima, compromiso profesional, clima institucional, valoración social, capital cultural, salarios, estímulos, incentivos, carrera profesional, evaluación del desempeño (p. 14).

En Colombia, la profesión docente en los niveles de educación básica y media está reglamentada por dos decretos que funcionan simultáneamente, el Decreto 2277 (1979) y el Decreto 1278 (2002), en el primero no existía un concurso de méritos como el actual, lo cual no quiere decir que no había un sentido de meritocracia, ya que se necesita de ciertos 
niveles de formación y los requisitos contemplados en el Decreto 259 (1981) para el ingreso. Por lo tanto, se puede considerar que este proceso era menos riguroso que el actual para la selección de los docentes al servicio del Estado. Por el contrario, en el Decreto 1278 (2002) el ingreso a la carrera docente está determinado mediante el concurso de méritos. Actualmente el ingreso a la carrera docente se realiza bajo el Decreto 1278 (2002). A pesar de los ajustes en materia salarial docente en los últimos años, estos siguen siendo inferiores comparados a otros trabajadores del Estado en este sentido Vaillant (2006) afirma:

La remuneración docente parece estar condicionando cada vez más el reclutamiento a la profesión. En efecto, como se indica en algunos estudios recientes, la docencia se ha transformado en una profesión que no paga lo suficiente como para atraer a los mejores candidatos, porque las estructuras de aumento salarial en la carrera son significativamente peores a las de otras profesiones. Esto hace que aquéllos que ingresan en los institutos de formación tengan, de promedio, peor historial educativo que otros estudiantes que acceden a otros estudios más valorados socialmente (p. 126).

Las afirmaciones anteriores, son claramente evidentes a final de año cuando se entregan los resultados de las pruebas de Estado SABER 11, la mayoría de los estudiantes que obtienen resultados bajos optan por elegir una carrera de licenciatura donde tienen más oportunidades de ser admitidos por una universidad pública, mientras que quienes tienen los recursos para estudiar en una universidad privada $u$ obtienen un buen resultado en dichas pruebas se decantan por otros estudios como medicina, ingenierías, etc. La profesión docente por sus bajos salarios y por el poco reconocimiento de la sociedad hoy en día no es atractiva para los jóvenes.

Con base en lo anterior, Saavedra y Forero (2018) sostienen que gran parte de los estudiantes con bajo desempeño académico en el bachillerato terminan estudiando carreras de educación, y, por otro lado, el contexto socioeconómico de las familias de dichos estudiantes no les permite tener los recursos para que sus hijos puedan ingresar a universidades acreditadas en alta calidad.

Debido a las situaciones anteriormente presentadas, se observa un panorama preocupante, ya que existe un fuerte vínculo entre la calidad de la educación y el desempeño que el docente tiene dentro del aula de clases, por lo que el gobierno de Colombia, a través del Ministerio de Educación, ha tratado de implementar diferentes mecanismos para subsanar estos problemas que deberían minimizarse desde la misma formación inicial, entre ellos el Programa Todos a Aprender PTA.

Mediante el programa antes mencionado, PTA, se pretende realizar un mejoramiento en la calidad de la educación para jóvenes y niños de las instituciones educativas focalizadas, evidenciado en el mejoramiento de los resultados de las pruebas externas SABER cómo uno de sus objetivos principales, este programa se comenzó a implementar en el año 2013 en las instituciones que presentaron resultados más bajos en las pruebas SABER y con altos índices de deserción (Rodríguez y Pantoja, 2018, p. 15).

Cabe destacar que en el marco de PTA, se orientan bajo unos componentes estratégicos, como lo son el pedagógico en donde se busca mejorar las prácticas de aula en las áreas de matemáticas y lenguaje, para que los estudiantes puedan desarrollar sus competencias básicas, en esta se tiene en cuenta el uso pedagógico de las pruebas SABER. Un componente de formación situada en donde hay un diálogo entre docentes y tutores sobre las 
necesidades, problemáticas y se hace un acompañamiento dentro del aula de clases. Un componente de gestión educativa en donde se busca que cada actor que interviene en el proceso educativo asuma responsabilidades y actúen de forma coordinada. Por último, un componente de condiciones básicas el cual garantiza que la institución educativa cuente con los elementos básicos para trabajar y las autoridades locales suministren programas importantes como el de alimentación escolar, así como las alianzas público-privadas que se puedan establecer.

Dentro del programa PTA, también se han incluido unas competencias socioemocionales, para ello ha dispuesto desde el ministerio de educación unas guías para los diferentes grados escolares, estas competencias MEN (2017), las define como: "aquellas que incluyen no solo el desarrollo de procesos cognitivos o mentales sino también áreas afectivas como la conciencia y gestión emocional, de relacionamiento con otros y de proyección hacia la sociedad" (p. 5). Por lo que ayudarán a mejorar las relaciones con los demás, bajar la agresión, lo que se traducirá en un mejor rendimiento académico y toma de decisiones en la vida. Partiendo de todos los elementos anteriores que componen la estrategia de PTA, se pueden presentar de la siguiente manera:

Para el PTA es importante la estrategia de administrar mejor el tiempo en el salón de clase y el clima de aula, que mejora la calidad educativa y muchos menos tiempos muertos, lo que implica tener en cuenta los niveles de aprendizaje de los diferentes integrantes del grupo, los intereses, gustos, habilidades para poder desarrollar una planeación más detallada y efectivas en el desarrollo (Barreto et al., 2016 citados en Rodríguez y Pantoja, 2018, p. 298).

Finalmente, mencionar el aspecto de llevar a cabo una evaluación formativa en donde se tenga en cuenta las características de los estudiantes, identificar que les ayudó a aprender, porque no aprenden con algunas estrategias, metodologías implementadas en el aula de clases, que permita reflexionar sobre los aprendizajes (autoevaluación, heteroevaluación). Ahora bien, el gobierno nacional ha dispuesto una evaluación anual a los docentes del sector público, que sirve en gran medida para cumplir los objetivos que se trazan en otros programas como PTA, puesto que los capacitadores rendirán un informe a los coordinadores, rectores, funcionarios de las secretarias de educación que servirán de evidencias a la hora de evaluar el desempeño del docente.

Esta evaluación de desempeño que se realiza anualmente, por lo general a final de cada año, según el calendario especificado por cada una de las secretarias de educación y busca medir de forma sistemática el desempeño de los docentes y directivos docentes de acuerdo a las funciones que este debe realizar en la institución educativa, si se están cumpliendo los objetivos planteados al inicio del año escolar dentro del área del conocimiento, trabajo en equipo, sus relaciones con la comunidad educativa, entre otras, que permitirán identificar las fortalezas y debilidades para así retroalimentar mediante acciones de mejora o estímulos.

En Colombia, la Ley 715 (2001) ha sido reglamentada mediante diversos decretos, uno de ellos es el Decreto 1278 (2002), que a su vez se implementa mediante el Decreto 3782 (2007), las cuales rigen la evaluación anual de desempeño laboral de los docentes y directivos. Esta "permite verificar el quehacer profesional de los educadores identificando fortalezas y aspectos de mejoramiento" (MEN, 2011, p. 1). 
La evaluación de desempeño, también ha supuesto una división entre docentes que pertenecen al estatuto 2277 (1979) y 1278 (2002), estos últimos son los que se les aplica este mecanismo, y por esto, en el caso de los docentes, la mayoría de veces son sobre quienes recaen muchas de las actividades programadas en las instituciones educativas, entre ellas actos cívicos y proyectos de aula, ya que a final de año tendrán que mostrar evidencias de todas las actividades realizadas durante el año, lo que supone muchas veces conflictos entre compañeros, los cuales tiene que manejar sabiamente los directivos docentes de la institución.

La Federación Colombiana de Trabajadores de la Educación (FECODE), ha manifestado su profundo rechazo ante este tipo de evaluaciones, ya que colocan en perjuicio la estabilidad laboral del docente, esto debido a que, si se obtiene una calificación por debajo del $60 \%$ dos años consecutivos, se retira al docente del magisterio, en este sentido afirma que:

La política de evaluación se ha caracterizado como "punitiva" y "extorsiva", orientada a resultados sin tener en cuenta los procesos, el entorno y las circunstancias en que trabajan los docentes. La introducción de los conceptos de participación y concurrencia en la evaluación de desempeño deja abierta la posibilidad de intervención de actores sin formación y sin experiencia que puede dar lugar a persecuciones e injusticias que distorsionen su sentido (Pulido, 2007, p. 43).

Aún sigue vigente la evaluación de desempeño, la cual se hace necesaria, afortunadamente cuando hay desacuerdos sobre la calificación del evaluado, se puede acudir a una instancia superior para conciliar. Sin embargo, hay otros aspectos donde se han realizado acuerdos entre el sindicato FECODE y el Ministerio de Educación, es así que en el año 2019, el gobierno se compromete a mejorar el servicio de salud del magisterio, definición de tiempos para llegar a la consecución de un proyecto de estatuto único docente, nivelación salarial para los años 2020, 2021 y 2022, así como el incremento del porcentaje de la bonificación pedagógica que a partir del año 2021 hacia adelante será del 19\% de lo que devenga el docente, entre otros acuerdos pactados.

Por otro lado, se encuentra la problemática relacionada con la capacitación de los docentes a nivel de postgrados, algunas de la razones es la escasa oferta de maestrías y doctorados en nuestro país y aún más que apliquen a los contextos en donde se desenvuelve el docente, aparte de los costos que son elevados si se tiene en cuenta el dinero que este devenga, he allí donde el gobierno mediante diversas estrategias tiene que coadyuvar, para que docentes y directivos docentes puedan acceder a este tipo de estudios, otorgando subsidios y garantizando a través de la oferta de la universidad pública, estudios de este nivel a bajos costos para los maestros. En este año 2020, el Ministerio de Educación en plena pandemia COVID-19, en el día del Maestro da a conocer la plataforma Contacto Maestro, la cual hace parte de las estrategias de formación a las que pueden acceder docentes y directivos docentes, con acceso a cursos, diplomados, especializaciones, maestrías y doctorados. Se hacía necesario y de vital importancia, como lo expresa Buitrago (2018) al respecto:

Es muy importante que los programas de maestría y doctorado que se ofertan en el país mantengan y mejoren sus niveles, y que los maestros y directivos docentes que se forman en ellos asuman la investigación como una alternativa de desempeño profesional y como una posibilidad idónea para conocer y reflexionar sobre la cotidianidad y las necesidades de los contextos educativos, y orientar las acciones educativas, pedagógicas y didácticas que se requieren. 
En este sentido, es importante señalar que las especializaciones en el país fueron durante muchos años muy importantes y relevantes, pero que poco a poco, en muchos casos, fueron perdiendo calidad, rigor y pertinencia, y que, de manera desafortunada, muchos maestros se dedicaron a coleccionar un gran número de ellas, las cuales no siempre se vieron reflejadas en su quehacer docente (p. 5).

Teniendo en cuenta lo anterior, si el gobierno apoya a los docentes económicamente para tales estudios, es pertinente que se haga un seguimiento en cuanto a los proyectos que se generen en las instituciones educativas.

\section{El problema de las desigualdades sociales entre escuelas}

Otro problema recurrente, es referente a la disparidad en cuanto a infraestructura, recursos humanos y físicos en las diferentes escuelas del país, equilibrar estas condiciones requiere de una gran inversión por parte de las autoridades locales y nacionales. Muchos de los niños y jóvenes que asisten a clases llegan a los colegios con hambre, porque sus familias carecen de recursos para proporcionar alimentos, es muy difícil adquirir conocimientos en estas condiciones, habitan en barrios cuyos centros de salud están alejados, existen pandillas con líneas imaginarias que impiden el libre tránsito de la gente, drogadicción, mientras que, por otro lado, hay estudiantes que viven en contextos donde existen una gama de servicios que posibilitan de mejor forma el aprendizaje. En este sentido Roemer (1998) afirma:

Imagine usted un partido de fútbol entre dos equipos, el equipo rojo y el equipo azul. El juego, sin embargo, tiene la siguiente particularidad: el campo está inclinado a favor del equipo azul. Para llegar al arco contrario, los jugadores del equipo rojo no solo tienen que superar a los jugadores rivales, sino que deben hacer un esfuerzo extraordinario para correr hacia arriba y anotar en el arco contrario. Los del equipo azul, en cambio, se desgastan menos y pueden anotar más fácilmente (p. 71).

La anterior metáfora del campo de juego inclinado, se utiliza para referirse a "ciertas condiciones que determinan la suerte de las personas y que están por fuera de su control. Esas condiciones, son las circunstancias, entre las que se encuentran los genes, los antecedentes familiares, la cultura y en general, el medio social" (García, Espinosa, Jiménez y Parra, 2013, p. 7). Se puede inferir entonces que dos estudiantes de distintas escuelas con las capacidades, destrezas, habilidades, uno de ellos podría tener mejores condiciones para acceder a una educación de calidad, que el otro estudiante dependiendo el contexto donde se desenvuelve. En este sentido, el Banco Mundial (2008) afirma que:

Las características familiares, como los ingresos y la educación de los padres, son importantes para el aprendizaje escolar en Colombia y pueden ser particularmente influyentes en el desarrollo del alfabetismo y en determinar la escuela a la que asisten los alumnos (p. 2).

En relación a lo anterior, la OECD (2016), afirma que "Las desigualdades comienzan a temprana edad; muchos niños desfavorecidos nunca van a la escuela, o no empiezan a tiempo o asisten a instituciones de menor calidad. Las diferencias resultantes en términos de nivel de estudios alcanzado son abismales" (p.16). De manera que el Estado debería garantizar una educación de calidad desde los niveles iniciales y esto en gran medida se 
consigue implementando la jornada única y los tres grados en este nivel antes de llegar a la primaria, actualmente existe solo uno que es el grado transición que es garantizado por el gobierno nacional en las instituciones públicas del país. Se puede evidenciar que el artículo 18 de la Ley 115 (1994) -Ley general de educación-, el cual dice: "El nivel de educación preescolar de tres grados se generalizará en instituciones educativas del Estado o en las instituciones que establezcan programas para la prestación de este servicio", pero esto no ha ocurrido, es decir el Estado no ha cumplido, aquí es donde comienza la fragilidad del sistema educativo colombiano. Esto mismo se extrapola a los compromisos en la educación básica y media y ni qué decir del profundo rezago de los colegios privados de los estratos más bajos, es así como García et al. (2013) afirman que:

Los resultados del estudio de Bogotá indicaron que los estudiantes de colegios privados de clase alta (no todos los colegios privados son de clase alta) obtienen casi siempre mejores resultados en las pruebas del Icfes, mientras que los colegios oficiales, a donde van los estudiantes de clase media-baja y baja, obtienen puestos mediocres o malos. Eso significa que en Bogotá no solo hay un sistema educativo que ofrece niveles de calidad diferenciales según la clase social, sino que este impone, de hecho, una especie de apartheid educativo: los ricos estudian con los ricos y los pobres con los pobres (p. 13).

Habría que decir también que "los niños, en especial aquellos provenientes de hogares vulnerables, llegan a la escuela primaria con déficits cognitivos muy grandes que constituyen una fuente importante de fracaso escolar" (Fundación Santillana, 2016). Debe quedar bastante claro la importancia de comenzar a hacer cumplir las leyes, establecer prioridades y en educación es evidente que el nivel preescolar es una de ellas.

Bien, pareciera por todo lo anterior que unas bases sólidas en los primeros años de escolaridad son trascendentalmente fundamentales, si no se tienen entonces estos niños. En tal sentido la OECD (2016) afirma que "deben esforzarse al máximo para progresar a un ritmo aceptable, tienen que repetir años o desertar del todo".

Todo esto parece confirmar que, existen muchas familias de bajos recursos en especial en las zonas rurales (corregimientos, veredas) alejadas de las cabeceras municipales, población indígena, no logran acceder a una educación de calidad y las escuelas están muy lejanas y no se cuentan con los medios de transporte o los recursos económicos para hacer uso de ellos, lo cual hace que estas dificultades al cabo de un tiempo de estudio estos estudiantes y su núcleo familiar decidan abortar el proceso educativo para dedicarse a labores del campo o trabajos mal remunerados, esta situación la expresa Delgado (2014) en la cual afirma que: "de cada 100 estudiantes que ingresan al sistema educativo en la zona rural, 48 culminan la educación media, mientras que en las áreas urbanas lo hacen 82 estudiantes" (p. 3).

Si bien es cierto, que la educación pública adolece de muchos problemas como algunos de los mencionados a lo largo de este artículo, es importante resaltar que ha tenido algunas mejoras en cuanto a calidad y cobertura que son innegables, lo cual se ve reflejado en el incremento de estudiantes matriculados en las escuelas públicas en relación con las privadas en los últimos años. En este sentido, en la capital de Colombia se muestran unos datos interesantes al respecto: 
La experiencia de Bogotá es ilustrativa en este sentido. La ciudad implementó durante varias administraciones un conjunto de reformas tendientes a mejorar la calidad y eficiencia de su sistema educativo público, lo cual ha llevado a un rápido crecimiento de la matricula oficial, con el consecuente aumento en la participación de la educación pública del 54\% al 66\% entre los años 2002 y 2011 (Delgado, 2014, p. 5).

Las comunidades ven los cambios que tienen las instituciones educativas, en cuanto a su infraestructura, la cual incluye sus instalaciones, los salones, laboratorios, salas de informática, servicios adicionales como alimentación escolar, además de un cuerpo docente bien capacitado, además de la obtención de buenos resultados en las pruebas de Estado, le significan una alta demanda de padres de familia que quieren que sus hijos ingresen a estas instituciones educativas. En los últimos años la brecha se ha cerrado entre las escuelas privadas de estratos bajos con respecto a las instituciones educativas públicas, se espera que progresivamente se alcancen altos estándares por lo que se necesitan grandes esfuerzos económicos y compromiso de todos los actores que hacen parte del proceso educativo.

\section{Evaluación de los aprendizajes en los estudiantes}

La evaluación es sin duda uno de los elementos más importantes dentro del aula de clases, y les permite a los diferentes actores en el ámbito escolar tomar los correctivos necesarios en búsqueda de la mejora continua, con el fin llevar un proceso de formación del estudiante en el campo intelectual y en todas sus dimensiones que lo caracterizan como ser humano. Actualmente, la evaluación del aprendizaje en Colombia, a través del decreto 1290 de 2009 (Montes, Alarcón y Romero, 2019) está definida como:

Normatividad vigente en materia de la evaluación y promoción de los estudiantes de los niveles de básica y media del país, permite a los Establecimientos Educativos de manera autónoma, definir el sistema de evaluación e incorporarlo al sistema educativo, con lo cual también pueden definir el tipo de evaluación, modelos, y escala de valoración y criterios de promoción, lo que ha constituido mayor diversidad de enfoques y ponen en manos de la escuela asuntos ampliamente criticados por el magisterio colombiano, tales como la promoción automática y la amplia flexibilidad (p. 5).

Por otra parte, las instituciones educativas deben adecuar su Proyecto Educativo Institucional -PEI- en concordancia con el decreto 1290 de 2009 en lo que concierne al aspecto evaluación, para ello se deberán generar reuniones con la comunidad educativa en donde se hagan análisis, reflexiones y se lleguen a acuerdos que permitan integrar la evaluación según el modelo pedagógico por el cual se orienta la institución educativa, el tipo de evaluación que se debe aplicar a los estudiantes, la elaboración de instrumentos que permitan consignar los resultados de esa evaluación, la reestructuración de los diferentes planes de área en base a ello, para poder determinar los contenidos y estrategias más adecuadas para que se lleve el proceso de enseñanza-aprendizaje. La evaluación debe ser integral y de acuerdo a las características del grupo de estudiantes, reconociendo las particularidades de cada uno de ellos. En este sentido, Murillo e Hidalgo (2015) afirman: 
Para lograr una verdadera valoración y reconocimiento de todas las personas sin exclusión, es necesario que los docentes promuevan una evaluación sensible a las diferencias culturales de sus estudiantes. Ello implica, por una parte, que las estrategias de evaluación se adapten a las diferencias culturales de cada estudiante; pero también que los docentes sean conscientes de los valores, cultura e ideologías imperantes en el currículum oculto (p. 6).

La necesidad de establecer revisiones y establecer nuevas normas en educación, como el citado decreto 1290 que regula la evaluación en educación en Colombia, se hace bajo análisis de resultados de las diferentes pruebas internas e internacionales, un ejemplo de esta última es la prueba PISA que "evalúa no solo los conocimientos de los estudiantes, sino sus capacidades para aplicarlos en situaciones cotidianas" (ICFES, 2018), dejó evidenciado un rezago en lectura, matemáticas y ciencias, con respecto a la mayoría de los países que hacen parte de la OCDE, como se plantea en los resultados del año 2018 a continuación:

Los estudiantes de Colombia obtuvieron un rendimiento menor que la media de la OCDE en lectura (412 puntos), matemáticas (391) y ciencias (413), y su rendimiento fue más cercano al de los estudiantes de Albania, México, la República de Macedonia del Norte y Qatar (OECD, 2019, p. 1).

Debido a estos resultados el gobierno nacional, ha implementado algunas iniciativas, como las emprendidas por el Ministerio de la Cultura mediante el Plan de Lectura y Escritura "Leer es mi cuento", busca mejorar los hábitos de lectura y que los habitantes de nuestro país vuelvan su mirada a los libros. Para esto se pretende dotar a las escuelas públicas de libros a los que puedan acceder los estudiantes, así mismo fortalecer las bibliotecas públicas. Con esta iniciativa, se espera mejorar los niveles de comprensión, afianzar la capacidad de poder expresar los pensamientos e ideas a través de la escritura, ya que es preocupante los resultados obtenidos en las pruebas nacionales e internacionales de los estudiantes de nuestro país, como lo expone OECD (2019) de la siguiente forma:

En Colombia, 50\% de los estudiantes alcanzaron al menos el Nivel 2 de competencia en lectura (media de la OCDE: 77\%). Como mínimo, estos estudiantes pueden identificar la idea principal en un texto de extensión moderada, encontrar información basada en criterios explícitos, aunque algunas veces complejos, y pueden reflexionar sobre el propósito y la forma de los textos cuando se les instruye explícitamente para hacerlo (p. 2).

Igualmente, en el área de matemáticas, se han adoptado experiencias de otros países, como por ejemplo el Modelo Singapur, el cual, según Turizo, Carreño y Crissien (2019), "su objetivo es el de fortalecer las habilidades de los niños en relación a la solución de problemas y no al desarrollo de algunos contenidos los cuales, la mayoría de veces, carecen de significado para los estudiantes", es importante este tipo de iniciativas que se apalancan en sistemas con mejores prácticas evidenciados en los buenos resultados obtenidos en las pruebas estandarizadas internacionales. En el área de Matemáticas, es indispensable si tenemos en cuenta los resultados obtenidos, que son los siguientes:

Cerca de 35\% de los estudiantes de Colombia alcanzaron el Nivel 2 o superior en matemáticas (media de la OCDE: 76\%). Como mínimo, dichos estudiantes son capaces de interpretar y reconocer, sin instrucciones directas, cómo representar matemáticamente una situación (simple) (por ejemplo, comparar la distancia total entre dos rutas alternativas o convertir precios a una moneda diferente) (OECD, 2019, p. 2). 
Desde luego, que exige el compromiso de docentes, directivos docentes en vincular este tipo de estrategias en sus clases, en la ciudad de Barranquilla se hizo la capacitación a los docentes de transición y primaria buscando que los docentes profundizaran sus conocimientos y didácticas sobre las matemáticas, buscando un mejor aprendizaje y competencias en los estudiantes.

\section{Impacto de la calidad educativa en una nación}

Hoy en día los países desarrollados demandan calidad en la prestación de sus servicios y productos cualesquiera que sean, así mismo es aplicable al sector educativo en donde se espera que en las escuelas capaciten personas que se enfrenten a los desafíos de forma exitosa, que cuente con las herramientas para salir adelante a lo largo de su vida en los diferentes ámbitos tanto a nivel personal, familiar, social, etc. La calidad de la educación debería ir de la mano con el crecimiento de un país, situación que según Barrera-Osorio, Maldonado y Rodríguez (2012) no sucede en la región y lo expresan así: "es interesante notar que la relación entre calidad de la educación y crecimiento es más débil para los países de América Latina que para otros países del mundo".

Siguiendo esta misma línea de ideas, la calidad de educación permite que las personas estén mejor preparadas, trabajen de forma eficiente, logren constituir organizaciones, empresas que desarrollen productos y/o servicios de forma rápida, de calidad y con bajos costos, lo cual generan ventajas competitivas frente a otros en el mercado internacional, pero para que esto ocurra se deben tener los conocimientos y las competencias necesarias. Es allí donde se genera una sensación de calidad de vida de los ciudadanos, como afirma Camargo (2001) lo siguiente:

El camino hacia la calidad apunta a construir una vida digna a los ciudadanos, a través de la obtención de bienestar y equidad. En estos aspectos claves se ha puesto el énfasis reciente de la contribución de la educación al desarrollo económico y social equitativo del país (p. 16).

La realidad colombiana, muestra un panorama poco esperanzador en desarrollo humano, el cual se recrudece si se analiza la realidad de cada departamento. De acuerdo con el último informe del que habla el Programa de las Naciones Unidas para el DesarrolloPNUD (Organización de las Naciones Unidas-ONU, PNUD, 2016), por ejemplo, tasas de pobreza por encima del 50\% como Chocó, Cauca y la Guajira (son mayoritariamente poblados por minorías indígenas y afrodescendientes) mientras otros tienen tasas de 10\% como Bogotá.

Dos tercios de los departamentos del país resultaron por debajo del puntaje promedio nacional en las Pruebas Saber 11 en 2017; sumado a esto, la periferia se encuentra considerablemente por debajo, mientras que los departamentos del centro y la región Andina están ligeramente por debajo o por encima del promedio. Este último es el caso de Bogotá, Santander, Cundinamarca, Boyacá, Norte de Santander, Meta, Valle del Cauca y Quindío (Meisel y Granger, 2019, p. 7).

Esto demuestra según Rojas (2018), que hay una desigualdad regional importantísima, e implica que tenemos capas de la población que están siendo excluidas de los beneficios 
del desarrollo económico, que no pueden alcanzar niveles básicos para potencializar sus capacidades y así lograr el bienestar (p. 10).

En relación con la calidad de la educación, es evidente el rezago de los departamentos de la periferia del país con respecto a los departamentos del centro y la región Andina. Aunque no hay consenso en cuanto a los determinantes de estas disparidades, algunas de las razones recaen sobre las desigualdades en la formación docente y la carencia de instituciones que aseguren una inversión eficaz del gasto público (Meisel y Granger, 2019, p. 4).

Para mejorar estos aspectos, Corredor (2019) hace una serie de recomendaciones en relación con tres factores: personales, sociales y escolares, en cuanto al primero lo describe de la siguiente forma:

En relación a los factores personales las regiones con los mejores resultados han incluido en sus programas de gobierno acciones para el desarrollo de las competencias en una política congruente, que se complementan con programas de formación de docentes y programas escolares de mejoramiento de las instituciones educativas. Sin embargo, las regiones con los resultados más bajos no han sido constantes en desarrollar estrategias para el desarrollo de las competencias básicas, ciudadanas o tecnológicas (p. 134).

Lo anterior, coloca de manifiesto que en algunos departamentos de nuestro país, existen muchos docentes que no cuentan con la formación docente pertinente, sobre todo las poblaciones más apartadas como corregimientos y veredas a muchas horas de distancia de las cabeceras municipales, existen muchas localidades como que estas, que se encuentran enmarcadas en zonas de conflicto armado, y por otro lado las carencias que se viven en la población en términos de servicios públicos y atención en salud, hacen que los mejores maestros no quieran postularse a estos cargos y los que laboran allí por concurso, piden traslado al poco tiempo de estar en esos territorios.

Ahora bien, con respecto al segundo aspecto que son los factores sociales, hacen alusión a las condiciones, sobre todo de tipo económico y las posibilidades de estudiar en su contexto, es por ello que se deben buscar alternativas para incentivar a los estudiantes no solo en el último año, sino durante toda su vida escolar lo cual contribuirá a que se motive a estudiar y obtener buenos resultados, que le permitirá acceder a universidades, obtener becas, entre otros. En este sentido, Corredor (2019) afirma que:

Los factores sociales que también se relacionan con los resultados de la calidad de la educación media, han sido más relevantes para las regiones que han logrado avances en los resultados en las pruebas académicas, estas regiones tienen en común el apoyo a estudiantes para educación superior, becas, subsidios, lo cual constituye una oportunidad para seguir el proceso de formación y al mismo tiempo una motivación a obtener mejores resultados individuales para acceder a las ayudas ofrecidas por las gobernaciones (p. 134).

Finalmente, para fortalecer la calidad de la educación, hay que tener en cuenta los aspectos institucionales, en donde se debe propender que el docente adelante investigaciones en el aula, igualmente desde las directivas la revisión y seguimiento del proyecto educativo institucional en lo que concierne al modelo educativo y si se están llevando a cabo las estrategias metodológicas adecuadas en relación a este. Corredor (2019) lo describe de la siguiente manera: 
En relación a los factores escolares se observó que la iniciativa de mejorar la calidad de la educación ha estado enfocada a la formación de docentes, para desarrollar programas de investigación, lectura y en los últimos años se ha incluido la implementación de la jornada única, el mejoramiento de la infraestructura y dotación escolar, y la implementación de una metodología en nuevos ambientes de aprendizaje, modelos educativos pertinentes y uso de tecnología (p. 130).

Algunos de los retos para mejorar la calidad educativa en Colombia, están orientados a mejorar en un trabajo mancomunado entre los diferentes actores que intervienen en el proceso educativo en nuestro país, desde el ministerio de educación con sus políticas educativas, las secretarías de educación en la destinación de los recursos a cada una de las instituciones y el seguimiento pertinente en cuanto a lo académico, convivencial, planta de personal docente, entre otras, la participación de los padres de familia y por último, toda la disposición de los docentes para utilizar todas las estrategias y metodologías eficaces para conseguir los mejores resultados y brindar a la sociedad un mejor ciudadano con principios y valores.

\section{Conclusiones}

Indudablemente, los esfuerzos realizados por Colombia han sido significativos en cuanto al perfeccionamiento de su sistema nacional de evaluación educativa, pero aún no es suficiente para alcanzar los estándares de calidad presentados en resultados obtenidos en las pruebas estandarizadas en relación con países que se encuentran en la OCDE.

Se confirma la existencia, puesta en marcha y pertinencia de algunos programas creados por el Ministerio de Educación para mejorar la calidad educativa en Colombia.

Se recomienda la creación de guías ilustrativas en cada área del conocimiento que se encuentren enmarcadas en los estándares básicos de competencias, que incluyan no solo aquellas que tradicionalmente se colocan énfasis como matemáticas, lengua castellana, sino que abarquen un espectro más grande que incluyan educación física, artística, tecnología e informática, entre otras.

Destaca la brecha abismal entre la calidad educativa del área urbana con respecto al área rural que se evidencia en los resultados de las pruebas nacionales, luego esto se ve reflejado en el impacto que esto genera en los departamentos que tienen una población eminentemente rural como Chocó, Cauca, entre otras, las cuales no avanzan en los diferentes campos de la economía lo que no permite mejorar la calidad de vida de sus habitantes.

Se sugiere entonces, crear espacios de discusión desde cada una de las secretarias de educación de los entes territoriales en departamentos y municipios priorizados según los resultados de las pruebas nacionales con el fin de abordar las problemáticas que se presentan en las comunidades, así como adaptar currículos pertinentes según las necesidades del contexto.

Mediante esta revisión, se pone de manifiesto la necesidad de incrementar los recursos para la capacitación docente mayormente en estudios de postgrado a nivel de maestrías y doctorados, considerando que deben culminar con un producto (tesis), orientada a resolver un problema de su institución educativa o contexto.

Es necesario identificar diversas estrategias de evaluación que permitan mirar el funcionamiento del sistema educativo, entre ellos aspectos relevantes como la dirección efectiva 
de las instituciones educativas, el uso eficiente de los recursos, el desempeño docente. Igualmente, se deberán hacer esfuerzos en dotación de tecnología y priorizar mejorar la infraestructura escolar, así como implementar la jornada única que permitirá que los estudiantes estén mayor tiempo en las escuelas y fortalezcan sus conocimientos, y de paso los aleja muchas veces de un contexto residencial y familiar que no son muy sanos. Para que todos estos cambios se den, debe haber una verdadera transformación vinculada a grandes reformas educativas con un gran compromiso del gobierno y la sociedad que logren generar políticas de Estado que configuren un mejoramiento en la calidad de la educación en el país.

\section{REFERENCIAS}

Arancibia, V. (1997). Los sistemas de medición y evaluación de la calidad de la educación. OREALC/1997/PI/H/3. [Online]. Recuperado de http://unesdoc.unesco.org/ images/0018/001836/183651s.pdf

Ayala-García, J. (2015). Evaluación externa y calidad de la educación en Colombia. Documentos de Trabajo sobre Economía regional, (17) [Online]. Recuperado de http://www. banrep.gov.co/docum/Lectura_finanzas/pdf/dtser_217.pdf

Banco Mundial. (2008). La calidad de la educación en Colombia: un análisis y algunas opciones para un programa de política. Documento 43906. [Online]. Recuperado de http:// documentos.bancomundial.org/curated/es/522681468026421049/pdf/439060ESW0P10 610Box342010B01PUBLIC1.pdf

Barrera-Osorio, F., Maldonado, D. y Rodríguez, C. (2012). Calidad de la educación básica y media en Colombia: diagnóstico y propuestas. Serie documentos de trabajo, (126). [Online]. Recuperado de http://www.urosario.edu.co/urosario_files/7b/7b49a017-42b046de-b20f-79c8b8fb45e9.pdf

Barreto, Y., Valdovinos, O., Vicente, U., Salazar, C., Flores, P. y Gómez, A. (2016). Medición del tiempo efectivo de la clase de educación física y su impacto en el gasto calórico en escolares de nivel primaria del municipio de Colima, México. Sportis, 3(1), 34-49. https://doi.org/10.17979/sportis.2017.3.1.1766

Black, P. \& Wiliam, D. (1998). Assessment and Classroom Learning. Assessment in Education, 5(1), 7-74. https://doi.org/10.1080/0969595980050102

Braun, H. \& Kanjee, A. (2006). Using Assessment to Improve Education in Developing Nations. In: H. Braun, A. Kanjee, E. Bettinger \& M. Kremer, Improving Education, Through Assessment, Innovation, and Evaluation. Cambridge: American Academy of Arts and Sciences.

Buitrago, R. (2018). Transformaciones sociales y educativas desde procesos investigativos. Praxis \& Saber, 9(21), 9-20. https://doi.org/10.19053/22160159.v9.n21.2018.8922

Camargo, M. (2001). Un marco teórico sobre la calidad de la educación, basado en lectura documental. [Tesis de grado]. Universidad de la Sabana, Chía, Colombia. Recuperado de http://studylib.es/doc/7173520/un-marco-te\%C3\%B3rico-sobre-la-calidad-de-laeducaci $\%$ C3\%B3n

Campos, C. y Fajardo, M. (2015). La calidad de la educación, una problemática colombiana. Revista Edu-Física.com, 7(16), 19-25. Recuperado de http://revistas.ut.edu.co/index. php/edufisica/article/view/1007/783 
Córdoba, F. (2006). La evaluación de los estudiantes: una discusión abierta. Revista Iberoamericana de Educación, 39(7), 1-8. Disponible en https://rieoei.org/RIE/article/ view/2537

Corredor, N. (2019). Factores de la calidad educativa desde una perspectiva multidimensional: Análisis en siete regiones de Colombia. Plumilla Educativa, 23(1), 121-139. https://doi.org/10.30554/plumillaedu.1.3350.2019

Crooks, T. (1988). The Impact of Classroom Evaluation Practices on Students. Review of Educational Research, 58(4), 438-481. https://doi.org/10.3102/00346543058004438

Chacón, L. (2019). Calidad educativa: una mirada a la escuela y al maestro en Colombia. Revista Educación y Ciudad, (36), 35-49. https://doi.org/10.36737/01230425. V1.N36.2019.2120

DANE. (2018). Censo Nacional de Población y Vivienda 2018. [Online]. Recuperado de https://www.dane.gov.co/files/censo2018/infografias/info-CNPC-2018total-nal-colombia.pdf

Díaz-Barriga, F. y Hernández, G. (2002). Estrategias docentes para un aprendizaje significativo: una interpretación constructivista (2 ed.). México, D.F.: McGraw-Hill.

Delgado, M. (2014). La educación básica y media en Colombia: retos en equidad y calidad. Informa final. [Online]. Disponible en http://hdl.handle.net/11445/190

Fundación Santillana. (2016). Construyendo una educación de calidad: un pacto con el futuro de América Latina Comisión para la Educación de Calidad para Todos. [Online]. Recuperado de http://1m1nttzpbhl3wbhhgahbu4ix.wpengine.netdna-cdn.com/wp-content/uploads/2016/08/Construyendo-una-educacio\%CC\%81n-de-calidad-WEB.pdf

García, M., Espinosa, J., Jiménez, F. y Parra, J. (2013). Separados y desiguales. Educación y clases sociales en Colombia. Bogotá, D.C.: Reino de los países bajos. Recuperado de https://www.dejusticia.org/wp-content/uploads/2017/04/fi_name_recurso_591.pdf

ICFES. (2018). Pruebas Internacionales. [Online]. Recuperado de https://www.icfes.gov. co/documents/20143/237489/Presentacion\%20evento\%20de\%20socializacion\%20-\%20 pisa\%202018.pdf

ICFES. (2012). Estudios sobre calidad de la educación en Colombia. [Online]. Recuperado de https://www.icfes.gov.co/documents/20143/232527/Estudios+sobre+calidad+de+la+e ducacion+en+Colombia+2012.pdf

Meisel, A. y Granger, A. (2019). ¿Atrapados en la periferia? Brechas de calidad en la educación en Colombia: Pruebas Saber 11 (2000-2018). En, Uninorte, Serie Documentos N. 36. Barranquilla: Universidad del Norte. Recuperado de https://www.uninorte.edu.co/documents/611589/0/publicacion/7171b636-c771-43c9-8b7f-474b341d3709?version=1.0

Montes, A., Alarcón, A. y Romero, Z. (2019). Enfoque de la evaluación en la Educación Básica y Media en la región Caribe Colombiano. Tendencias y realidades. Revista Espacios, 40(9), 3. Recuperado de http://www.revistaespacios.com/a19v40n09/a19v40n09p03.pdf

Murillo, F. e Hidalgo, N. (2015). Dime cómo evalúas y te diré qué sociedad construyes. Revista Iberoamericana de Evaluación Educativa, 8(1), 5-9. Consultado de https:// revistas.uam.es/index.php/riee/article/view/2972/3192

OECD. (2019). Programa para la Evaluación Internacional de Alumnos (PISA). [Online]. Recuperado de https://www.oecd.org/pisa/publications/PISA2018_CN_COL_ESP.pdf 
OECD. (2018). OCDE Revisión de Recursos Escolares. [Online]. Recuperado de http://www. oecd.org/education/school/OECD-Reviews-School-Resources-Summary-Colombia-Spanish.pdf

OECD. (2016). Revisión de políticas nacionales de educación. La educación en Colombia. [Online]. Recuperado de http://www.mineducacion.gov.co/1759/articles-356787_recurso_1.pdf

ONU. PNUD. (2016). Objetivos de Desarrollo Sostenible, Colombia Herramientas de aproximación al contexto local. [Online]. Recuperado de https:/www.undp.org/content/dam/ colombia/docs/ODM/undp-co-ODSColombiaVSWS-2016.pdf

Pulido, O. (2007). La Federación Colombiana de Educadores (FECODE) y la lucha por el derecho a la educación. El Estatuto Docente. Buenos Aires: Laboratorio de Políticas Públicas. Recuperado de http://biblioteca.clacso.edu.ar/ar/libros/argentina/lpp/fecode. pdf

REDUCA. (2015). Situación educacional en Colombia: una mirada desde los principales indicadores educativos. [Online]. Recuperado de http://www.educacion2020.cl/sites/default/files/informe_reduca_colombia.pdf

República de Colombia. Congreso de la República. (21 de diciembre de 2001). Por la cual se dictan normas orgánicas en materia de recursos y competencias de conformidad con los artículos 151, 288, 356 y 357 (Acto Legislativo núm. 01 de 2001) de la Constitución Política y se dictan otras disposiciones para organizar la prestación de los servicios de educación y salud, entre otros. [Ley 715]. Diario Oficial No 44.654.

República de Colombia. Congreso de la República. (8 de febrero de 1994). Ley general de educación. [Ley 115]. Diario Oficial No. 41.214.

República de Colombia. MEN. (2017). Estrategia de formación de competencias socioemocionales en la educación secundaria y media. [Online]. Recuperado de https://www. mineducacion.gov.co/1759/articles-385321_recurso.pdf

República de Colombia. MEN. (2016). Colombia, la mejor educada en el 2025. Líneas estratégicas de la política educativa del Ministerio de Educación Nacional. [Online]. Recuperado de http://www.mineducacion.gov.co/1759/articles-355154_foto_portada.pdf

República de Colombia. MEN. (2011). Evaluación anual de desempeño de docentes y directivos docentes. Directiva ministerial sobre evaluación anual de desempeño de docentes y directivos docentes. [Online]. Disponible en http://www.mineducacion.gov.co/1759/ w3-article-246098.html

República de Colombia. MEN. (2009). Organización del Sistema Educativo. Guia 33. [Online]. Recuperado de http://www.mineducacion.gov.co/1621/articles-205294_archivo_pdf.pdf

República de Colombia. MEN. (20 de junio de 2002). Estatuto de Profesionalización Docente. [Decreto 1278]. Diario Oficial 44.840.

República de Colombia. Presidencia de la República. (2 de octubre de 2007). Por el cual se reglamenta la evaluación anual de desempeño laboral de los servidores públicos docentes y directivos docentes que se rigen por el Decreto-ley 1278 de 2002. [Decreto 3782]. Diario Oficial No. 46.769. 
República de Colombia. Presidencia de la República. (6 de febrero de 1981). Por el cual se reglamenta parcialmente el Decreto Extraordinario 2277 de 1979, en lo relacionado con inscripción y ascenso en el Escalafón. [Decreto 259]. Diario Oficial No. 35.703.

República de Colombia. Presidencia de la República. (14 de septiembre de 1979). Por el cual se adoptan normas sobre el ejercicio de la profesión docente. [Decreto 2277]. Diario Oficial No. 35.374.

Robalino, M. (2005). ¿Actor o Protagonista? Dilemas y responsabilidades sociales de la profesión docente. Revista Prelac, (1), 7-23. Recuperado de http://unesdoc.unesco.org/ images/0014/001446/144666s.pdf

Rodríguez, L. y Pantoja, A. (2018). La formación situada del "programa todos a aprender” en Boyacá. Revista Interamericana, 12(1), 295-317. Disponible en https://revistas. usantotomas.edu.co/index.php/riiep/article/view/4908

Roemer, J. E. (1998). "Igualdad de oportunidades". Isegoría, (18), 71-87. https://doi. org/10.3989/isegoria.1998.i18.146

Rojas, S. (2018). ¿Se puede hablar de equidad en el sector educativo colombiano? Revista Científica General José María Córdova, 16(23), 125-143. https://doi. org/10.21830/19006586.286

Saavedra, V. y Forero, D. (2018). Los 10 pasos para ser Colombia la Mejor Educada en 2025. [Online]. Recuperado de http://www.anif.co/sites/default/files/investigaciones/20180627_informe_final_-_premio_lcsa.pdf

Turizo, L., Carreño, C. y Crissien, T. (2019). El Método Singapur: reflexión sobre el proceso enseñanza - aprendizaje de las matemáticas. Pensamiento Americano, 12(23) 183199. Disponible en http://publicaciones.americana.edu.co/index.php/pensamientoamericano/article/view/255

UNESCO. (2008). Reflexiones en torno a la evaluación de la calidad educativa en América Latina y el Caribe. [Online]. Disponible en https://unesdoc.unesco.org/ark:/48223/ pf0000177648

Vaillant, D. (2006). Atraer y retener buenos profesionales en la profesión docente: políticas en Latinoamérica. Revista de Educación, 340, 117-140. Recuperado de http://www. revistaeducacion.educacion.es/re340/re340_05.pdf

Zambrano, M. (2015). Alcances e inconsistencias del Índice Sintético de Calidad Educativa (ISCE), diseñado y aplicado por el Ministerio de Educación de Colombia en el año 2015. Working paper. Bogotá, D.C.: Fundación IQ Matrix. Recuperado de https://studylib.es/ doc/5444350/alcances-e-inconsistencias-del-indice-sintetico-de-calida...

Jhan Carlos Herrera Pérez es Ingeniero de Sistemas, Especialista en Ingeniería de Software, Magister en ingeniería Telemática y Doctor en Educación. Participó en un concurso de méritos y ahora es docente de tecnología e informática en propiedad del Distrito de Barranquilla, en su institución es líder en proyectos de tipo pedagógico con apoyo de la tecnología, acompañando a los directivos docentes. https://orcid.org/0000-0003-1158-3494 